\title{
Chemical decaging
}

Researchers design a chemical decaging strategy for studying protein function in cells.

Small-molecules are ideal tools for manipulating and observing protein function in living cells, but developing specific and potent probes is a major challenge. Alternative strategies to knock down protein function exist but such experiments can run into roadblocks if even a small proportion of the protein is still active. In contrast, in a gain-of-function study, even very low enzyme activation efficiency is usually enough to turn on a signaling pathway. This may yield more mechanistic insights, explains Peng Chen of Peking University in China.

One type of gain-of-function approach involves the use of photocleavable 'caging' groups to turn off functional amino acids in proteins. When hit with light, the caging group is stripped away, restoring native protein activity. A handful of photocleavable unnatural amino acids are available that can be site-specifically incorporated into proteins in living cells using genetic code expansion methods. But the ultraviolet light required to trigger 'decaging' can cause surface-receptor internalization, altered intracellular signaling and cytotoxicity.

Chen and his colleagues now provide an alternative chemical decaging strategy, one that adds to the protein function manipulation toolbox and may provide advantages over lightbased decaging in many situations. "The toughest challenge we met was to find the proper bioorthogonal protection group and deprotection reagents (the caging and decaging pair), which can be used on intact proteins inside living cells," says Chen. "Although many deprotection methods have been developed for amino acids and peptides, most of them have to be carried out under harsh reaction conditions that are not suitable for biological applications." After extensively combing the chemical literature for an appropriate reaction, the researchers settled on a palladium catalyst-mediated depropargylation reaction.

But first, the team needed to show that decaging was robust. Initially, they optimized the decaging reaction in vitro, showing that it was efficient and did not cause any damage to the

\section{STEM CELLS}

\section{EMBRYONIC MATCHMAKING}

A nuclear-transfer technique informed by cell-cycle synchronization could simplify generation of therapeutic stem cells.

By inserting the nucleus from a mature cell into an oocyte from which the nuclear material has been removed, one can derive an embryo that retains the genetic makeup of the donor. Such cloning via somatic cell nuclear transfer (SCNT) could potentially offer a ready source of patient-specific embryonic stem cells (ESCs) for regenerative medicine applications.

Low success rates and a limited understanding of the cellular factors that drive reprogramming render this method as much an art as a science, however, and some researchers have concluded that this nuclear reprogramming may only be a transient property of the unfertilized egg. New findings from Shoukhrat Mitalipov and colleagues at Oregon Health \& Science University now indicate that the window is wider than expected (Kang et al., 2014).

"For a very long time, it was thought that right after fertilization this activity declines sharply," Mitalipov says. "But we always wondered if this cytoplasmic activity is really unique to unfertilized oocytes or whether other cell types have the same activity." In 2013, his team became the first group to successfully achieve SCNT with human cells (Tachibana et al., 2013) after years of failed attempts. One of the secrets of their success was ensuring that both the donor nucleus and recipient oocyte were at compatible stages of the cell cycle, and the researchers wondered whether careful cell-cycle 'matching' might also enable nuclear transfer with post-fertilization recipient cells.

They carefully monitored stage-specific cell-cycle indicators as single-cell mouse zygotes developed into two-cell embryos. "You could use drugs to block them at a certain cell-cycle stage, but this usually has a detrimental effect, and so we just monitored the zygotes," says Mitalipov. "When they first cleaved, that's when we started the timer." With this timetable in hand, they attempted to synchronize the transplantation of nuclei from fetal 


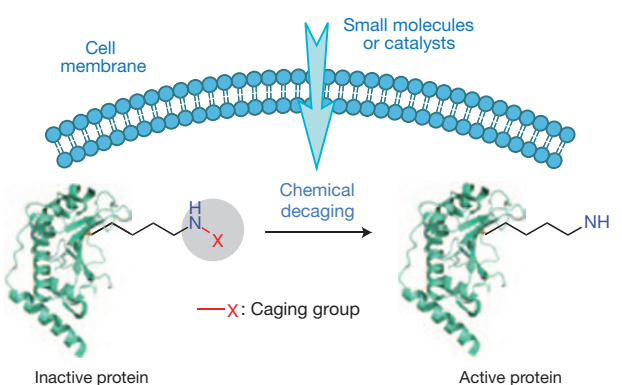

Chemical decaging of an unnatural amino acid using a palladium catalyst enables restoration of native protein function. Figure from Li et al., Nature Publishing Group. protein GFP. They then moved the reaction in vivo, decaging a fluorescent small molecule and GFP in mammalian cells, demonstrating that the palladium catalysts could enter living cells and that the decaging reaction was not toxic and was reasonably efficient.

Chen's team then truly put the method to the test by focusing on OspF, a phosphothreonine lyase that is secreted into host cells by pathogenic Shigella microbes. Once in the host cell, OspF irreversibly dephosphorylates mitogenactivated protein kinases such as phosphorylated Erk. The team introduced propargyl-lysine at position 134 in OspF's active site and expressed the protein in mammalian cells. Following decaging, they observed a restoration of OspF's dephosphorylation function. They tagged OspF and Erk in turn with GFP to follow their intracellular localization; OspF remained in the nucleus after decaging, but dephosphorylated Erk did not, suggesting a possible mechanism for OspF's virulence in host cells.

Though it remains to be tested, the chemical decaging strategy is likely to be general and may find use in tissues and possibly even animals. Chen points out that tools for genetically incorporating other unnatural propargyl-caged residues into proteins are already available. "This, in conjunction with the general applicability of the palladium-mediated deprotection chemistry, should allow our chemical decaging strategy to be expanded to other amino acids," he says.

\section{Allison Doerr}

\section{RESEARCH PAPERS}

Li, J. et al. Palladium-triggered deprotection chemistry for protein activation in living cells. Nat. Chem. $\mathbf{6}$, 352-361 (2014).

skin cells or ESCs into cells derived from interphase two-cell embryos (I2Cs).

The matching made a difference-nuclear transfer with close donor-recipient synchronization yielded embryos with a success rate roughly equivalent to that of standard SCNT with oocytes. In contrast, skewing the timing by just 30 minutes to an hour reduced the success rate by more than half. Mitalipov's team subsequently injected ESCs isolated from these embryos into naturally derived embryos from a different mouse strain. These experiments yielded healthy chimeric mice that also passed the donor genomic material to their offspring, an outcome confirming that I2C-derived ESCs were fully integrated into the embryo during development.

The researchers also obtained a few viable fetuses after implanting female mice with embryos derived entirely from ESC nuclei transferred into I2Cs. However, the same experiments failed for I2Cs that had received skin cell nuclei. Mitalipov hypothesizes that the more 'mature' nuclei were incompletely reprogrammed and failed to form the placental tissue that supports early fetal development. "This is one of the earliest lineages to form from the embryo, and for some reason they were always somewhat defective," he says. This could also be problematic with donor nuclei taken from adult somatic cells, which are even more tightly 'locked' into their developmental state.

However, Mitalipov's interest is in stem cell production rather than reproductive cloning, and his team's success in establishing pluripotent ESCs from I2Cs with skin cell nuclei suggests that this goal is within reach. His next objective is work with slightly 'older' recipient cells, namely, existing ESC lines, which would obviate the need for donor eggs or embryos. "We would have an unlimited source of pluripotent cells whose cytoplasm can be used to reprogram somatic cells," he says, "although we still have some technical issues that remain as roadblocks to testing this hypothesis." Michael Eisenstein

\section{RESEARCH PAPERS}

Kang, E. et al. Nuclear reprogramming by interphase cytoplasm of two-cell mouse embryos. Nature doi:10.1038/nature13134 (26 March 2014).

Tachibana, M. et al. Human embryonic stem cells derived by somatic cell nuclear transfer. Cell 153, 1228-1238 (2013). 